\title{
Improving the management of Acute Kidney Injury in a District General Hospital: Introduction of the DONUT bundle
}

\author{
Anisha Bhagwanani, Rory Carpenter, Aqeelah Yusuf
}

King's Mill Hospital, Sherwood Forest NHS Trust

\begin{abstract}
The NCEPOD report (2009) on Acute Kidney Injury (AKI) found $20 \%$ of post-admission AKIs were avoidable and only $50 \%$ of AKI care was considered 'good'. The DONUT bundle comprises of six interventions aimed at improving the management of AKI.

Baseline data was collected prospectively using the biochemistry eAlert system, identifying 50 patients with Stage 1 AKI over a two week period. Management was assessed 24 hours after the eAlert using a standardised proforma. After data analysis, a DONUT sticker was introduced within the Emergency Admissions Unit, providing an efficient method of recording interventions in the notes. Education sessions outlining the DONUT bundle and stickers were delivered via Foundation Program teaching, along with summary flash cards. A re-audit assessed these interventions.
\end{abstract}

Of the initial cohort $(n=50)$, only $8 \%$ of cases had all components of the care bundle completed. Following introduction of the education programme and AKI sticker, re-audit showed a rise in full compliance to $17 \%(n=42)$. Only $7 \%$ of cases used the AKI sticker but where it was used, there was $100 \%$ compliance with the bundle.

In conclusion, AKI management is sub-standard. An education program and the use of a simple sticker can improve management. Further education regarding $\mathrm{AKI}$ is needed and work is ongoing to improve compliance with sticker use.

\section{Problem}

Acute Kidney Injury (AKI) has recently gained significant publicity as a major cause of inpatient morbidity and mortality. AKI can be easily identified by a reduced urine output and/or deteriorating biochemistry and is often reversible if detected early. Poor recognition and management of these patients can lead to worsening renal function and eventual hyperkalaemia, metabolic acidosis and death.

The NCEPOD report: Adding Insult to Injury [1] identified $20 \%$ of post-admission AKIs to be preventable and only $50 \%$ of AKI management to be "good". The report identified poor assessment of risk factors for $\mathrm{AKI}$ with $33 \%$ of patients deemed to have had inadequate investigations. There was poor recognition of acute illness, hypovolaemia and sepsis. The report highlighted deficiencies in AKI management at a national level and produced a series of key recommendations. These include screening all emergency admissions for $\mathrm{AKI}$ and postgraduate education about the recognition and management of AKI.

The relative risk of AKI mortality at King's Mill Hospital, Sutton-InAshfield, stood at 215.7 in October 2012, notably higher than other local trusts. We suspected this may have been due to poor identification and management of these patients. Our quality improvement project was designed to identify areas of poor knowledge and management and design solutions to address them.

\section{Background}

Acute Kidney Injury, previously known as acute renal failure, is a sudden loss of renal function. AKI can be classified as Stage 1-3 according to the degree of oliguria or the rise in creatinine level [2], with higher stages being associated with poorer outcomes. It has been shown that there is a broadly linear relationship between the severity of $A K I$ and mortality risk [3]. AKI is a prevalent illness with an estimated $5-20 \%$ of critically ill patients experiencing an episode of AKI during their illness [4].

Causes of AKI can be divided into pre-renal, renal and post-renal. More specifically these include hypovolaemia, sepsis, obstruction of the urinary tract, toxicity from drugs or contrast agents and intrinsic renal diseases such as glomerulonephritis. Pre-renal causes account for the majority of cases. Good fluid balance management is therefore integral to the management of AKI, for which we refer the reader to the recent NICE guidelines on IV Fluid therapy [5].

The theory behind care bundles is that when several evidencebased interventions are grouped together in a single protocol, it will improve patient outcome [6]. The use of care bundles has been shown to improve healthcare quality outcomes in many cases when performed collectively and reliably [7].

The DONUT care bundle comprises of a useful mnemonic to recall first-line interventions in the management of all AKI stages:

D - Dehydration (All patients with an abnormally low systolic blood 
pressure $<100 \mathrm{mmHg}$ should be given an initial fluid bolus of $250 \mathrm{ml}$ of crystalloid and response assessed)

O - Obstruction (Bladder scan all patients within 24 hours and consider renal ultrasound to exclude obstruction)

$\mathrm{N}$ - Nephrotoxins (Stop nephrotoxic drugs )

U - Urine (Start a strict fluid balance chart and perform urinalysis on all patients)

T - Think Sepsis (Escalate according to Trust policy)

\section{Baseline Measurement}

We identified 50 patients with Stage $1 \mathrm{AKI}$ over a two week period in December 2012. Patients were identified prospectively via an electronic alert system which determined cases via an increase in creatinine level 1.5-2 times the baseline value. We assessed the management of these cases against the DONUT bundle using a standardised proforma, 24 hours after the initial alert. The cases identified were patients across both medical and surgical wards.

The ward with the largest number of AKI cases was the medical Emergency Admissions Unit ( $n=17)$.

D- Dehydration: Of 11 patients with an abnormally low systolic blood pressure less than $100 \mathrm{mmHg}, 3(27 \%)$ had a fluid bolus prescribed.

O - Obstruction: 9 patients (18\%) had a bladder scan performed and 8 patients $(16 \%)$ had a renal ultrasound scan.

$\mathrm{N}$ - Nephrotoxins: Of 37 patients identified to be on nephrotoxic drugs, 20 patients $(54 \%)$ had their medications reviewed.

U - Urine: 31 patients (62\%) had a fluid balance chart and 27 patients $(54 \%)$ had evidence of urinalysis results.

T- Think Sepsis: Separate work around the management of sepsis has been performed in the Trust and management of sepsis was therefore not included within the realm of this improvement project.

Only 4 patients (8\%) had full compliance with the care bundle.

See supplementary file: ds2212.pptx - "Fig1"

\section{Design}

The baseline data showed that all risk factors targeted by the DONUT bundle were being managed poorly, rather than identifying just one or two areas of sub-standard care. We suspected that this may be due to poor communication between health professionals and poor education around AKI management including correct IV fluid management. Anecdotal evidence suggested that junior doctors were reluctant to prescribe fluid boluses in elderly patients, particularly those with a history heart failure due to concerns regarding fluid overload.

We therefore decided that our ideas for change needed to include education and raising awareness of risk factors and important firstline management steps. The need for education was also highlighted by the absence of AKI teaching during the weekly Foundation Programme teaching sessions delivered to FY1 and FY2 doctors. Accordingly, we began by introducing the DONUT bundle to Foundation doctors during their timetabled teaching, as it is often these junior doctors who are responsible for the initial assessment and management.

Given that our baseline data demonstrated the largest number of $\mathrm{AKI}$ cases to be on the medical Emergency Admissions Unit (EAU), we targeted our interventions to this busy ward. We implemented our improvement ideas via 3 PDSA cycles with guidance from the hospital AKI group that comprised of a renal physician, consultant patient safety lead for the hospital, senior pharmacist and senior nurse.

\section{Strategy}

PDSA Cycle 1:

We introduced the DONUT bundle via FY1 and FY2 teaching sessions. During these teaching sessions, we distributed pocketsized DONUT flash cards, for the junior doctor to keep and use on the wards as a visual aide memoire. We also designed DONUT bundle posters to act as another visual reminder and these were displayed in each bay of EAU.

PDSA Cycle 2:

Following PDSA cycle 1, it was apparent that most people were still not using the DONUT care bundle for patients with AKI. Therefore we informed all doctors working on EAU about the DONUT bundle via email, and introduced a DONUT bundle sticker to be entered into the medical notes for all patients with AKI. This provided a reminder of the bundle at the point of care and provided an efficient method of recording the management of the AKI in the notes. We designed the sticker to be a series of tick boxes that would take a minimal amount of time to complete, as we recognised that there is already a significant amount of paperwork.

PDSA Cycle 3:

We continued to increase awareness of AKI and the DONUT bundle by presenting at the medical Grand Round meeting. During the implementation of these interventions, we continued to monitor compliance with the DONUT bundle on EAU, assessing the management of the AKI 24 hours after the eAlert was received.

\section{Results}

Successful implementation of a care bundle requires that all steps are completed collectively, for every patient. Therefore our process measure was the number of patients that had $100 \%$ compliance 
with the AKI bundle.

Baseline data collected in 50 patients with Stage $1 \mathrm{AKI}$ across all wards showed $8 \%$ of patients $(n=4)$ with full compliance with the DONUT bundle. 17 of these patients were on EAU and of these, $0 \%$ $(\mathrm{n}=0)$ had full compliance with the bundle.

Following the introduction of our interventions for change, we assessed the management of 42 patients with Stage 1 AKI on EAU. The re-audit showed an increase from $0 \%$ to $17 \%$ of patients $(n=7)$ with full compliance with the DONUT bundle on EAU. The AKI sticker was only used in $7 \%$ of patients $(n=3)$. However in all cases where the sticker was used, all stages of the bundle were completed. Fig. 2 shows the overall rates of compliance with the individual components of the care bundle.

See supplementary file: ds2260.pptx - "Fig2"

\section{Lessons and Limitations}

From our project we learned that simple interventions can result in improvement but that engagement and cooperation from all members of the healthcare team is vital for a successful outcome. Encouraging doctors to use the AKI sticker was our most significant challenge.

Our initial results suggest that the AKI sticker may be a useful tool for increasing compliance with the DONUT care bundle and the key recommendations produced by the NCEPOD report. However, the feedback that we received from doctors included that they forgot about the stickers, did not know where to find them or did not know what they looked like. Consequently, we recommend the production of a more permanent sticker/document that is available on all wards via the hospital intranet. We recommend introducing the DONUT bundle to the new doctors starting at the hospital each year in August as we found difficulties in introducing the bundle mid-way through the academic year.

We recognise that although there was a increase in overall compliance with the bundle, compliance with some individual components of the bundle actually decreased, most notably with respect to fluid balance charts and urinalysis. One limitation of our project was that education was limited to medical staff only. We recommend that this education is extended to include the nursing staff, as they are usually responsible for the accurate documentation of dipstick urinalysis and fluid balance. Successful management of these patients is dependent on the compliance of all members of the healthcare team.

Given that there was a poor rate of uptake of the AKI sticker, further work is required to assess its full potential for improving patient outcomes in AKI.

\section{Conclusion}

Poor management of Acute Kidney Injury has been identified at a national level, whilst our project has identified shortcomings at a local level. Given that basic clinical examination and routine tests can often help reverse the injury, increasing awareness and education about AKI management among those at the front line of care is an important priority that could directly improve patient outcomes.

We demonstrated that there was an overall poor assessment and management of risk factors for $\mathrm{AKI}$, rather than just one or two areas of particular concern. We have introduced a care bundle which provides a simple way of recalling basic interventions and provided an efficient way of recording these in the notes via the AKI sticker. The ongoing use of the care bundle will enable measures directly relating to patient outcomes to be assessed over a longer period of time.

Further work is required in the form of ongoing education to increase the cooperation of medical and nursing staff. A more permanent form of the AKI sticker is required to ensure that the intervention is sustainable.

\section{References}

1. National Confidential Enquiry into Patient Outcome and Death (NCEPOD). Adding Insult to Injury. A review of the care of patients who died in hospital with a primary diagnosis of acute kidney injury (acute renal failure), 2009. Available from: www.ncepod.org

2. Mehta R, Kellum J, Shah S, Molitoris B, Ronco C, Warnock $D$ et al. Acute Kidney Injury Network: report of an initiative to improve outcomes in acute kidney injury. Critical Care 2007 11: R31

3. London AKI Network. Why is acute kidney injury important? Available from: $w w w$.londonaki.net

4. Metnitz PG, Krenn CG, Steltzer H, Lang T, Ploder J, Lenz K et al. Effect of acute renal failure requiring renal replacement therapy on outcome in critically ill patients. Crit Care Med 2002; 30: 2051-2058.

5. National Institute for Health and Care Excellence. Intravenous Fluid Therapy, CG147. London: National Institute for Health and Care Excellence 2013. Available at: www.nice.org.uk/guidance

6. Fulbrook P, Mooney S. Care bundles in critical care: a practical approach to evidence-based practice. Nursing in Critical Care 2003; 8(6): 249-55.

7. Resar R, Griffin FA, Haraden C, Nolan TW. Using Care Bundles to Improve Health Care Quality. IHI Innovation Series White Paper 2012. Available at: www.IHI.org

\section{Declaration of interests}

Nothing to declare.

\section{Acknowledgements}

With thanks to Dr Simon Stinchcombe, Consultant Radiologist, for his guidance in mentoring this project. 